\title{
Refeeding syndrome: An overlooked condition?
}

\author{
Yuttiwat Vorakunthada MD, Passisd Laoveeravat MD, Wasawat Vutthikraivit MD, \\ Weerapong Lilitwat MD, FAAP, Ariwan Rakvit MD, FACG
}

\begin{abstract}
Refeeding syndrome (RFS) is commonly seen in different settings, particularly in the intensive care unit. However, it is not well recognized due to the lack of a standard definition. In general, hypophosphatemia is considered a diagnostic marker, but hypokalemia, hypomagnesemia, and shifts in thiamine and trace elements can also occur. Identification of high-risk patients is the key to prevention, especially in malnourished patients. Furthermore, introduction of oral, enteral, or parenteral nutrition in critically ill patients who have not received recent nutrition might lead to RFS. To date, there is no standard feeding protocol to prevent RFS. Therefore, awareness of RFS in high-risk patients, fluid and electrolyte treatment, and daily monitoring of clinical parameters are essential in the management of RFS.
\end{abstract}

Keywords: Refeeding syndrome, hypomagnesemia, hypokalemia, hypophosphatemia, malnourished patients, critically ill, nutrition

\section{INTRODUCTION}

Refeeding syndrome (RFS) represents a constellation of clinical symptoms of metabolic abnormalities in malnourished patients undergoing refeeding, whether orally, enterally, or parenterally. ${ }^{1}$ It is commonly described but often a neglected condition. Thus, health care providers should be vigilant in identifying high-risk patients and initiating prompt treatment. This article aims to provide a review of this overlooked condition, including pathophysiology, risk factors, prevention, and treatment.

To date, there is no consensus guideline in RFS definition. However, the National Institute for Health and Clinical Excellence (NICE) ${ }^{2}$ has proposed clinical criteria to identify patients at high risk. ${ }^{3,4}$

Corresponding author: Yuttiwat Vorakunthada Contact Information: Y.vorakunthada@ttuhsc.edu DOI: $10.12746 /$ swrccc.v6i23.466
Either the patient has one or more of the following:

- Body mass index $(\mathrm{BMI})<16 \mathrm{~kg} / \mathrm{m}^{2}$

- Unintentional weight loss $>15 \%$ in the past 3 to 6 months

- Minimal nutritional intake for $>10$ days

- Hypokalemia, hypophosphatemia, or hypomagnesemia before feeding

Or the patient has two or more of the following:

- $\mathrm{BMI}<18.5 \mathrm{~kg} / \mathrm{m}^{2}$

- Unintentional weight loss $>10 \%$ in the past 3 to 6 months

- Minimal nutritional intake for $>5$ days

- History of alcohol or drug abuse

Despite the identification of risk and prompt treatment with hypo-caloric nutritional therapy, some patients still develop RFS. ${ }^{3}$ In general, hypophosphatemia is considered a diagnostic marker for RFS, but one systematic review reported no consistent 
pattern of biochemical and chemical abnormalities in RFS. ${ }^{5}$ Also, hypophosphatemia can occur in other medical disorders. To date, recommendations for RFS are derived from only cohort and case series studies but not randomized controlled trials. ${ }^{6}$

\section{EPIDEMIOLOGY}

The exact prevalence of RFS is unknown due to lack of universally accepted definition. ${ }^{7}$ Diagnostic criteria have varied widely based on the institution. ${ }^{7}$ Nevertheless, the prevalence has been reported to range from $0.43 \%$ to $34 \%$, albeit a possible underestimation. ${ }^{3}$ Hence, it is a priority to recognize patients at risk before the initiation of feeding (Table 1). ${ }^{1,3,4,6}$

\section{Pathophysiology}

During starvation, the body's glycogen storage is depleted within one day, and the natural response is the transition to protein and fat metabolism. ${ }^{6}$ This metabolism is tightly regulated through hormonal mechanisms which decrease the level of insulin in the blood in contrast to the level of glucagon and growth hormone. This results in a catabolic state with lipolysis, proteolysis, gluconeogenesis, and production of ketone bodies. The release of fatty acids and

\section{Table 1. Patients at high risk for RFS}

- Eating disorder (e.g., anorexia nervosa, bulimia)

- Chronic alcoholism or drug abuse

- Bariatric surgery

- Elderly patient (e.g., > 70 years ago)

- Chronic infection (e.g., HIV)

- Dysphagia and esophageal dysmotility

- Malignancy

- Malabsorption syndrome (e.g., inflammatory bowel disease, short bowel syndrome, Cystic fibrosis, Celiac disease)

- Undernourishment (e.g., kwashiorkor, marasmus)

- Chronic antacids or diuretics use

- Uncontrolled diabetes

- Chronic liver disease

- Admission to Intensive Care Unit glycerol from adipocytes and amino acids (alanine) from myocytes provides essential substrates for gluconeogenesis. ${ }^{8}$ The rising level of ketone bodies, in turn, stimulates the brain tissue to utilize ketone bodies instead of glucose. ${ }^{4}$ The body also responds physiologically by inducing hypotension, bradycardia, and a reduction of basal metabolic rate (BMR) of up to $25 \%{ }^{9}$

During refeeding, the surge of insulin and depression of glucagon will drive potassium, magnesium, phosphate, glucose, and water into the cell, and there is a transition back to carbohydrate metabolism. Patients may have normal levels of routine laboratory results, but most electrolytes are intravascularly depleted due to prolonged starvation.

\section{Clinical implications AND PRESENTATIONS}

Refeeding syndrome involves a transcellular shift of electrolytes, vitamins, and fluid by hormonal and metabolic regulation. Clinical features of RFS arise from electrolyte derangements and rapid changes in body metabolism.

\section{HYPOPHOSPHATEMIA}

Phosphate is predominantly an intracellular anion that is tightly regulated in a concentration between 2.5 and $4.5 \mathrm{mg} / \mathrm{dl}$. It is essential for a wide range of intracellular processes, including glycolysis and oxidative phosphorylation for ATP generation, regulation of hemoglobin affinity for oxygen through the formation of 2,3-diphosphoglycerate, the maintenance of the functional integrity of cell membranes, acting as a secondary messenger system, and acid-base buffering. Hypophosphatemia can manifest as respiratory failure due to diaphragmatic weakness and cause prolonged mechanical ventilation.

\section{HYPOKALEMIA}

Potassium is found mainly in the cells, and the level is controlled tightly through the action of sodium-potassium ATPase. With refeeding, severe hypokalemia ensues due to insulin secretion that 
drive intracellular shift of potassium. Hypokalemia is also a marker for RFS. Potassium is essential for maintaining electrical neutrality and acid-base balance. Severe hypokalemia $(<2.5 \mathrm{mmol} / \mathrm{l})$ can cause cardiac arrhythmia, cardiac arrest, rhabdomyolysis, hepatic encephalopathy, severe muscular weakness, paralytic ileus, and refractory metabolic alkalosis.

\section{Hypomagnesemia}

Like potassium and phosphate, magnesium is primarily an intracellular divalent cation that is also depleted during RFS. It serves as a vital co-factor for enzymatic processes, such as oxidative phosphorylation and ATP production. Magnesium is required for maintaining cell membrane integrity and the structural integrity of genetic materials (DNA, RNA). Hypomagnesemia can precipitate seizures, cardiac arrhythmias, neuromuscular abnormalities, refractory hypokalemia, and hypocalcemia through decreases in parathyroid hormone function.

\section{HYPERGLYCEMIA}

Insulin release upon refeeding will suppress gluconeogenesis and promote lipogenesis. A cohort study has shown that administration of IV glucose before initiation of artificial nutrition support can precipitate RFS. ${ }^{10}$ As feeding continues, the circulating level of glucocorticoids subsequently increases, contributing to hyperglycemia. Prolonged hyperglycemia causes osmotic diuresis and dehydration, increases the risk of infection, and increases carbon dioxide production and hypercapnic respiratory failure in patients with pre-existing lung diseases. ${ }^{11}$

\section{THIAMINE AND OTHER VITAMIN DEFICIENCIES}

Like all water-soluble vitamins, thiamine is stored in a limited amount in the human body, and it is rapidly depleted in starvation. Thiamine is an essential coenzyme for carbohydrate metabolism. Thiamine deficiency can result in beriberi, lactic acidosis, Wernicke's encephalopathy, and Korsakoff's syndrome. Also, low levels of vitamin B6, B12, and folate are found in patients with RFS. ${ }^{6}$ These deficiencies can lead to anemia and neurological complications.

\section{SODIUM RETENTION AND FLUID IMBALANCE}

Refeeding will stimulate sodium and fluid retention as a secondary response to the intracellular shift of electrolytes from the action of insulin. This is essential for maintaining electrical neutrality in the extracellular compartment. Accumulation of fluid and decrease in renal excretion can lead to peripheral edema, fluid overload, acute kidney injury, and cardiac failure.

\section{TREATMENT}

Refeeding syndrome is managed by considering the possibility of its existence and by using the simple laboratory tests as described above. If the syndrome is suspected, the rate of feeding should be slowed down, and electrolytes should be replaced. In this review, we will focus on fluid balance, electrolyte imbalance, nutritional deficiency, and other parameters.

\section{FLUID BALANCE}

Fluid overload is a common complication of RFS, and therefore, assessment of intravascular fluid status is an essential step to guide fluid therapy to prevent congestive heart failure. ${ }^{4,6}$ Also, monitoring of serum sodium and daily fluid balances can help guide fluid treatment. To avoid fluid overload during necessary rehydration, we suggest administration of maintenance rates to achieve basic needs without causing fluid overload. Fluid and sodium should be further restricted in the presence of edema or congestive heart failure. In patients at high risk for cardiac decompensation, central venous pressure and cardiac rhythm monitoring should be considered.

\section{CORRECTION OF ELECTROLYTE IMBALANCE}

The best method of electrolyte replacement has not yet been determined, but intravenous supplementation in critically ill patients is recommended to avoid 
Table 2. Weight-based phosphorus replacement

\begin{tabular}{|ll|l|}
\hline \multicolumn{2}{|l|}{ Phosphorus level } & Replacement Regimen \\
\hline Mild & $2.3-3 \mathrm{mg} / \mathrm{dL}$ & $0.32 \mathrm{mmoL} / \mathrm{kg}$ \\
\hline Moderate & $1.6-2.2 \mathrm{mg} / \mathrm{dL}$ & $0.64 \mathrm{mmoL} / \mathrm{kg}$ \\
\hline Severe & $1.5 \mathrm{mg} / \mathrm{dL}$ & $1 \mathrm{mmoL} / \mathrm{kg}$ \\
\hline
\end{tabular}

Actual body weight, if $<130 \%$ of ideal body weight (IBW). Adjust body weight if $>130 \%$ IBW. Adjusted body weight $=$ IBW +0.25 (actual body weight - IBW).

issues with malabsorption. ${ }^{4,6}$ There are controversial recommendations regarding rates of electrolyte replacement in different studies.

\section{HYPOPHOSPHATEMIA}

There is no consensus on replacement regimen for hypophosphatemia. Potassium phosphate in normal saline solution has been commonly used but sodium phosphate is also available. Phosphorus replacement may be contraindicated in hypercalcemia because of the risk of metastatic calcification., ${ }^{3,4,6}$ The recommended dose is $15-30 \mathrm{mmol}$ of phosphate, which is adequate to replace severe hypophosphatemia. Also, a weight-based regimen has been shown to restore phosphate levels by day 2 and return them to normal levels within three days by Brown and colleagues (Table 2). ${ }^{12}$

\section{HyPOMAgNESEMIA}

Oral magnesium replacement can cause gastrointestinal tract disturbance; intravenous correction is preferred over the enteral route. Magnesium is typically given in the form of intravenous magnesium sulfate or magnesium chloride with a dose of $0.5 \mathrm{mmol} /$ $\mathrm{kg} /$ day over 24 hours, then $0.25 \mathrm{mmol} / \mathrm{kg} /$ day for five days. Screening for calcium level may be necessary because hypocalcemia can occur and requires replacement.

\section{HYPOKALEMIA}

The dose of replacement can be $20-40 \mathrm{mEq}$ for mild to moderate hypokalemia ( $\mathrm{K}: 2.5-3.4 \mathrm{mEq} / \mathrm{L})$. The dose of replacement should be increased to $1-1.5 \mathrm{mEq} / \mathrm{kg} /$ day in severe hypokalemia $(\mathrm{K}<2.5 \mathrm{mEq} / \mathrm{L})$. It is crucial to follow potassium levels approximately 2 hours post infusion to allow time for intracellular replenishment. Enteral supplementation is preferred due to safety and good bioavailability but has the side effect of gastrointestinal irritation. However, intravenous administration is feasible depending on rate and concentration. The peripheral administration should not exceed $10 \mathrm{mEq} / \mathrm{h}$ at a concentration of 40 to $80 \mathrm{mEq} / \mathrm{L}$. When using central lines, the rate may be increased to a maximum of $40 \mathrm{mEq} / \mathrm{h}$ at a maximum concentration of 80 to $120 \mathrm{mEq} / \mathrm{L}^{12}$

\section{VITAMIN SUPPLEMENTATION}

All vitamin deficiencies can occur in RFS, but thiamine deficiency is commonly found as a complication of refeeding. Patients can present with Wernicke's encephalopathy or Korsakoff's syndrome. According to the NICE guideline, the recommendation to supplement vitamin immediately, before, and during the first ten days of refeeding. ${ }^{2}$ The dose of each vitamin is suggested as follows: oral thiamine 200-300 mg daily, high potency vitamin B 1-2 tablets for three times daily, and a multivitamin once daily. Serum ferritin, vitamin B12, and folate concentration should be monitored. ${ }^{2}$ Regarding trace elements, including zinc, selenium, manganese, copper, chromium, the NICE guideline has recommended dosages for each trace elements for RFS.

\section{CALORIC REQUIREMENT}

Once RFS is diagnosed, the rate of feeding should be slowed down, and essential electrolytes should be replaced. ${ }^{4}$ Low caloric intake feeding can decrease the 6 month mortality rate by $60 \%$ of RFS patients on mechanical ventilators for more than seven days, compared to a high caloric intake group. ${ }^{13}$ This is also supported by the systematic review that showed caloric restriction with a protocol can improve mortality and survival time. ${ }^{7}$

Regarding the composition of the diet, high carbohydrate intake is more likely to cause RFS in contrast to the low amino acid intake. ${ }^{3}$ Current guideline recommends daily protein intake of approximately 
1.2 to $1.5 \mathrm{~g} / \mathrm{kg}$, or about $0.17 \mathrm{~g}$ nitrogen $/ \mathrm{kg}^{2,3,4}$ Moreover, very low-calories diet intake can result in an underfeeding syndrome with higher morbidity and mortality from insufficient energy intake.

\section{OTHER PARAMETERS}

Close monitoring of liver function and glucose is recommended in RFS. Hepatic dysfunction and hypo/ hyperglycemia have been reported. Of note, "rebound" hypoglycemia can occur when parenteral nutrition is stopped unless it is titrated down gradually. Infection, another complication, can occur in RFS. Monitoring of signs of localized infection and inflammatory markers are helpful in early detection.

\section{Prevention}

Identification of patient at risk is the critical point to prevent patients from developing RFS. Nutritional assessment before refeeding is required to assess the risk to develop RFS. These patients should start nutritional support at a maximum rate of $25 \mathrm{kcal} / \mathrm{kg} / \mathrm{day}$, increasing gradually to meet or exceed maximum needs by 4-7 days. However, in extreme patients with BMI less than $14 \mathrm{~kg} / \mathrm{m}^{2}$ or negligible intake for more than 15 days, the nutrition support would be only $5 \mathrm{kcal} / \mathrm{kg} / \mathrm{day}$. Continuous cardiovascular monitoring is warranted in this group and in patients who have a prior history of cardiac arrhythmias. Also, monitoring fluid balance, restoring circulatory volume, and frequent assessment of overall clinical status are also necessary.

The composition of diet and vitamin supplementation has been studied to prevent RFS. Low glucose and high fat diet is the best combination that can avert RFS because from the report of high glucose administration before feeding related to RFS. Oral thiamine (200-300 mg daily), vitamin B complex (strong 1-2 tablets for three times daily) or full dose of daily intravenous vitamin B preparation should be provided before and during the first ten days of feeding.

Daily electrolyte supplement containing potassium (2-4 mmoL/kg/day), phosphate $(0.3-0.6 \mathrm{mmoL} /$ $\mathrm{kg} /$ day), magnesium $(0.2 \mathrm{mmoL} / \mathrm{kg} /$ day IV, $0.4 \mathrm{mmoL} /$ $\mathrm{kg} /$ day oral) can help avoid metabolic complications from RFS. Pre-feeding correction of low plasma levels of electrolyte and minerals is unnecessary as the replacement of electrolyte before starting feeding can prolong the period of malnutrition. ${ }^{14}$

\section{NOVEL BIOMARKER FOR REFEEDING SYNDROME}

There is no reliable measurement to predict RFS. ${ }^{15}$ Leptin and insulin growth factor-1 (IGF1) are potential biomarkers. The refeeding index $(\mathrm{RI})$ was defined as the product of leptin and IGF1 divided by 2800 . Well-nourished patients will have the ratio of 1.0. The area under receiver operative characteristics curve (AUROC) for $a \geq 30 \%$ phosphate drop was highest in $\mathrm{RI}$ compared to IGF1 or leptin alone (AUROC $=0.75$, 0.64 , and 0.65 , respectively). The cut-off value of RI which can predict a significant decrease of phosphate is 0.19 with optimal sensitivity and specificity. Some other possible biomarkers to predict the development of refeeding hypophosphatemia are pre-albumin and ghrelin ${ }^{3}$, but more studies are needed to support the potential role of these markers.

\section{AREAS of RESEARCH AND DEVELOPMENT}

Regarding diagnosis, the diagnostic criteria and definition of RFS are the core area for the future research to establish the diagnosis and facilitate research. In line with a definite diagnosis, early detection and prediction will be the keys to prevent and monitor patients closely. Potential biomarkers will help identify patients at risk. Also, establishing a protocol to advance feeding per calories intake needs to be studied.

\section{ConcLusion}

Refeeding syndrome is an important under recognized condition that can develop in critically ill patients. Abnormalities of electrolytes, such as hypophosphatemia, hypomagnesemia, hypokalemia and fluid balance, are associated with substantial morbidity and mortality. Keys to prevention in highrisk patients are to gradually advance low glucose, high-fat caloric intake, supplement electrolytes and 
vitamin, and monitor clinical and laboratory parameters. Due to lacking a universally accepted definition of RFS, more prospective research is warranted.

Article citation: Vorakunthada $Y$, Laoveeravat $P$, Vutthikraivit W, Lilitwat W, Rakvit A. Refeeding syndrome: an overlooked condition? The Southwest Respiratory and Critical Care Chronicles 2018;6(23):4-9

From: The Department of Internal Medicine at Texas Tech University Health Sciences Center in Lubbock, TX (YV, PL, WV, AR) and the Department of Pediatric Critical Care Medicine, University of lowa Hospitals and Clinics, lowa City, IA (WL).

Submitted: $1 / 31 / 2018$

Accepted: $3 / 29 / 2018$

Reviewer: Scott O'Banion, Pharm D

Conflicts of interest: none

This work is licensed under a Creative Commons Attribution-ShareAlike 4.0 International License.

\section{REFERENCES}

1. Crook MA, Hally V, Panteli JV. The importance of the refeeding syndrome. Nutrition (Burbank, Los Angeles County, Calif) 2001;17(7-8):632-7.

2. National Institute for Health and Clinical Excellence (NICE). Nutrition support in adults. Clinical guideline CG32 2006. Last updated August 2017. https://www.nice.org.uk/ guidance $/ \operatorname{cg} 32$

3. Crook MA. Refeeding syndrome: problems with definition and management. Nutrition (Burbank, Los Angeles County, Calif) 2014;30(11-12):1448-55.

4. Mehanna HM, Moledina J, Travis J. Refeeding syndrome: what it is, and how to prevent and treat it. BMJ (Clinical research ed) 2008;336(7659):1495-8.
5. Skipper A. Refeeding syndrome or refeeding hypophosphatemia: a systematic review of cases. Nutrition in clinical practice: official publication of the American Society for Parenteral and Enteral Nutrition 2012;27(1):34-40.

6. Byrnes MC, Stangenes J. Refeeding in the ICU: an adult and pediatric problem. Current opinion in clinical nutrition and metabolic care 2011;14(2):186-92.

7. Friedli N, Stanga Z, Sobotka L, et al. Revisiting the refeeding syndrome: Results of a systematic review. Nutrition (Burbank, Los Angeles County, Calif) 2017;35:151-60.

8. Solomon SM, Kirby DF. The refeeding syndrome: a review. JPEN Journal of parenteral and enteral nutrition 1990;14(1): 90-7.

9. Marik PE, Bedigian MK. Refeeding hypophosphatemia in critically ill patients in an intensive care unit. A prospective study. Archives of surgery (Chicago, Ill: 1960) 1996;131(10): 1043-7.

10. Rio A, Whelan K, Goff L, et al. Occurrence of refeeding syndrome in adults started on artificial nutrition support: prospective cohort study. BMJ open 2013;3(1).

11. Inoue $T$, Doi $T$, Beppu $K$, et al. Warning to "Refeeding syndrome". Torsades de pointes and ventricular tachyarrhythmia with marked QT prolongation induced by acute glucose injection: report of two cases. International journal of cardiology 2012;156(2):222-4.

12. Parli SE, Ruf KM, Magnuson B. Pathophysiology, treatment, and prevention of fluid and electrolyte abnormalities during refeeding syndrome. J Infus Nurs 2014;37(3):197-202.

13. Olthof LE, Koekkoek W, van Setten C, et al. Impact of caloric intake in critically ill patients with, and without, refeeding syndrome: A retrospective study. Clinical nutrition (Edinburgh, Scotland) 2017.

14. Mehanna H, Nankivell PC, Moledina J, et al. Refeeding syndrome-awareness, prevention and management. Head \& neck oncology 2009;1:4.

15. Elnenaei MO, Alaghband-Zadeh J, Sherwood R, et al. Leptin and insulin growth factor 1: diagnostic markers of the refeeding syndrome and mortality. The British journal of nutrition 2011;106(6):906-12. 